\title{
ON THE LINEAR RELATIONS CONNECTING THE COMPONENTS OF THE DISCRETE WIGNER DISTRIBUTION IN THE CASE OF REAL-VALUED SIGNALS
}

\author{
C. Richard, R. Lengellé \\ Laboratoire de Modélisation et Sûreté des Systèmes \\ Université de Technologie de Troyes \\ 12 rue Marie Curie, BP 2060, 10010 Troyes cedex, France \\ E-mail: cedric.richard@univ-troyes.fr
}

\begin{abstract}
Recently, it was shown that information conveyed by the discrete Wigner distribution is highly redundant, linear relations connecting its time-frequency components. This means that every component of the discrete Wigner distribution can be expressed as a linear combination of the elements of a basis. This set of generators consists of particular time-frequency components of the distribution. However, up to now, this basis and the associated linear map that allows to entirely generate the representation have still not been characterized. In this paper, this problem is addressed in the case of real-valued signals. Results are illustrated by means of computer simulations. Finally, some extensions are pointed out.
\end{abstract}

\section{INTRODUCTION}

The Wigner distribution (WD) is a prominent tool that combines a temporal and a spectral analysis of signals by representing them over a joint time-frequency (TF) plane. Its usefulness partly derives from the fact it satisfies many desirable mathematical properties [1, 2, 3, 4]. For a practical implementation of $\mathrm{TF}$ analysis on a digital computer, the WD of a signal $\mathrm{x}$, which will be denoted $\mathrm{W}_{\mathrm{x}}$, must be reformulated in a discrete-time discretefrequency setting. It is clear that information conveyed by $\mathrm{W}_{\mathrm{x}}$ is redundant since the discrete WD derives $N \times N$ representations from $N$-sample signals. As far as we know, this redundancy was only studied in $[5,6]$, where it is shown that linear relations connect TF components of the discrete WD. This means that every component $\mathrm{W}_{\mathrm{x}}[n, m]$ can be expressed as a linear combination of the elements of a basis denoted $\mathscr{\delta}$. This set of generators consists of particular TF components $\mathrm{W}_{\mathrm{x}}[n, m]$. However, up to now, $\mathscr{S}$ and the associated linear map that allows to generate every TF component, which will be denoted $\mathrm{L}_{\mathscr{\delta}}$, have still not been absolutely characterized. The general subject of this work is the analytic characterization of $\mathcal{O}$ and $\mathrm{L}_{\delta}$ when $\mathrm{x}$ is a real-valued signal.
This paper is organized as follows. In Section 2, the number of linearly-independent TF components $\mathrm{W}_{\mathrm{x}}[n, m]$ of the discrete WD, denoted $N_{\mathscr{S}}$, is determined. Section 3 provides a necessary and sufficient condition satisfied by the $N_{\mathcal{S}}$ elements of $\mathcal{S}$. Then, the linear map $\mathrm{L}_{\delta}$ is characterized. Section 4 presents an example. Finally, some extensions are pointed out in the concluding Section 5.

\section{DETERMINATION OF $N_{\mathcal{S}}$}

Let $\mathbf{x}=(\mathrm{x}[0], \ldots, \mathrm{x}[N-1])^{T}$ be an element of a linear discrete-time signal space $\mathscr{X}$. In this work, we shall consider that $\mathscr{X}$ denotes the space of $N$-sample realvalued signals with $N$ an even integer. ${ }^{1}$ The discrete-time discrete-frequency WD of $\mathbf{x}$ is defined as

$\mathrm{W}_{\mathrm{x}}[n, m]=\sum_{k=1-N / 2}^{N / 2-1} \mathrm{R}_{\mathrm{x}}[n, k] \exp \left(-\frac{4 j \pi m k}{N}\right)$,

with $(n, m) \in\{0, \ldots, N-1\}^{2}$. Here, the autocorrelation function $\mathrm{R}_{\mathrm{x}}[n, k]$ is given by $\mathrm{R}_{\mathrm{x}}[n, k]=\mathrm{x}[n+k] \mathrm{x}^{*}[n-k]$ if $(n+k, n-k) \in\{0, \ldots, N-1\}^{2}, 0$ otherwise. From the definition (1), we define the maps $r$ and $w$ as

$\mathbf{x} \stackrel{r}{\longrightarrow} r(\mathbf{x})=\mathbf{r}_{\mathrm{x}}$ where $\mathbf{r}_{\mathrm{x}}=\left(\ldots, \mathrm{R}_{\mathrm{x}}[n, k], \ldots\right)^{T}$

$\mathbf{r}_{\mathrm{x}} \stackrel{w}{\longrightarrow} w\left(\mathbf{r}_{\mathrm{x}}\right)=\mathbf{w}_{\mathrm{x}}$ where $\mathbf{w}_{\mathrm{x}}=\left(\ldots, \mathrm{W}_{\mathrm{x}}[n, m], \ldots\right)^{T}$

such that $\mathbf{w}_{\mathrm{x}}=(w \circ r)(\mathbf{x})$. Let $\mathscr{R}$ and $\mathscr{\mathcal { W }}$ denote the spaces spanned by $r(\mathbf{x})$ and $(w \circ r)(\mathbf{x})$, respectively, i.e., that are given by

$\mathscr{R}=\left\{\mathbf{r}_{\mathrm{x}}: \mathbf{r}_{\mathrm{x}}=r(\mathbf{x}), \mathbf{x} \in \mathscr{Q}\right\}$

$\mathscr{W}=\left\{\mathbf{w}_{\mathrm{x}}: \mathbf{w}_{\mathrm{x}}=(w \circ r)(\mathbf{x}), \mathbf{x} \in \mathscr{X}\right\}$.

Since vectors $\mathbf{r}_{\mathrm{x}}$ and $\mathbf{w}_{\mathrm{x}}$ have finite numbers of components, there exist finite sets of linearly-independent vectors that are generators for $\mathscr{R}$ and $\mathscr{N}$, respectively. ${ }^{2}$ In this work, we shall define the dimension of $\mathscr{R}$ and $\mathscr{N}$, which will be denoted $\operatorname{dim}(\mathscr{R})$ and $\operatorname{dim}(\mathscr{W})$, as the

\footnotetext{
${ }^{1}$ Analogous results can be obtained in the case where $N$ is odd.

${ }^{2}$ Vectors generating $\mathscr{R}$ and $\mathscr{N}$ are not necessarily members of $\mathscr{R}$ and $\mathscr{W}$, respectively, since these spaces are not linear one.
} 


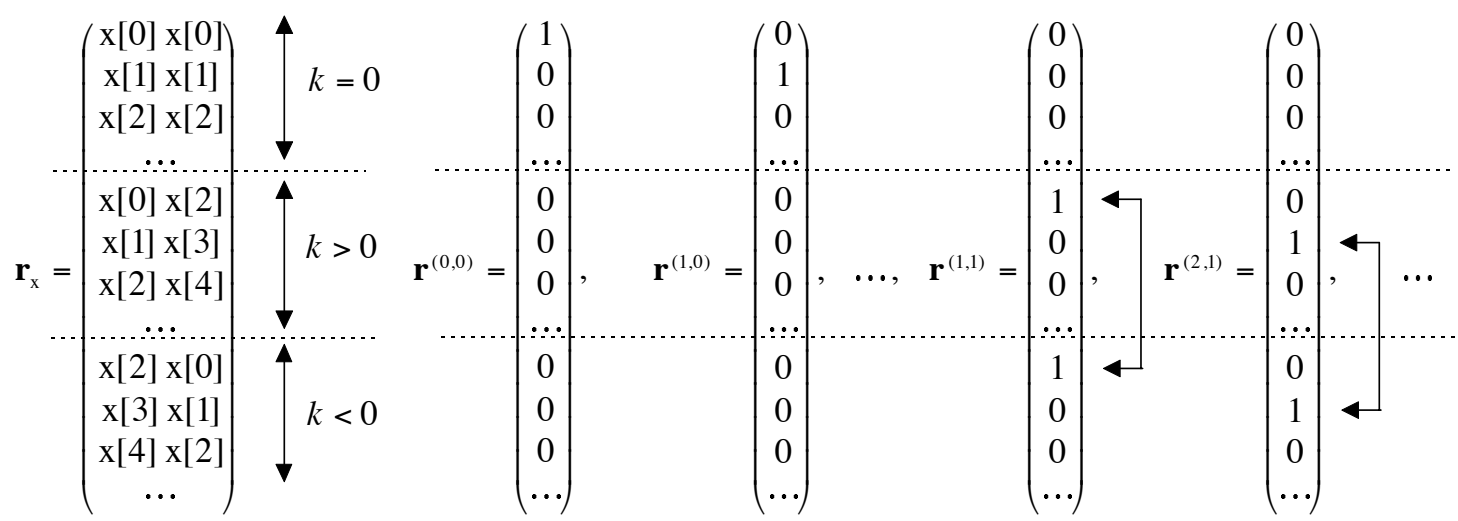

Figure 1. Construction of a set of orthogonal vectors that are generators for $\mathscr{R}$. Note that each vector $\mathbf{r}^{(n, k)}$ is associated with the cross-products $\mathrm{x}[n+k] \mathrm{x}^{*}[n-k]$ and $\mathrm{x}[n-k] \mathrm{x}^{*}[n+k]$.

minimum number of vectors required to generate these spaces. The reader will see at once that the number $N_{\mathscr{S}}$ of linearly-independent components of $\mathbf{w}_{\mathrm{x}}$ is in fact $\operatorname{dim}(\mathscr{W})[7,8,9]$. Since, given $n$, the WD is the Fourier transform of the autocorrelation function, $w$ is an isomorphism of the space $\mathscr{R}$ into $\mathscr{W}$. This implies $\operatorname{dim}(\mathscr{R})=\operatorname{dim}(\mathscr{W})=N_{\mathcal{S}}$. The next theorem provides $N_{\mathcal{S}}$.

Theorem 1. Let $\mathbf{x}=(\mathrm{x}[0], \ldots, \mathrm{x}[N-1])^{T}$ be a $N$-sample real-valued signal with $N$ an even integer. The number of linearly-independent TF components $\mathrm{W}_{\mathrm{x}}[n, m]$ of the discrete $W D$ is given by

$N_{\delta}=N^{2} / 4+N / 2$.

Proof. We leave it to the reader to verify that the only linear relations connecting the components of $\mathbf{r}_{\mathrm{x}}$ are those connecting $\mathrm{x}[n+k] \mathrm{x}^{*}[n-k], \quad k>0, \quad$ and $\mathrm{x}[n+k] \mathrm{x}^{*}[n-k], k<0$. It then follows from the set of generators for $\mathscr{R}$ proposed in Fig. 1 that

$$
\begin{aligned}
N_{\delta} & =\operatorname{card}\left\{\mathbf{r}^{(n, k)}\right\} \\
& =\operatorname{card}\left\{(n+k, n-k) \in\{0, \ldots, N-1\}^{2}, k \geq 0\right\} .
\end{aligned}
$$

Combining $0 \leq n+k \leq N-1$ and $0 \leq n-k \leq N-1$ gives $0 \leq k \leq n \quad$ if $\quad 0 \leq n \leq \operatorname{int}\{(N-1) / 2\}, \quad 0 \leq k \leq N-n-1$ otherwise. ${ }^{3}$ Since $N$ is even, we finally obtain

$N_{\mathcal{S}}=\sum_{n=0}^{N / 2-1}(n+1)+\sum_{n=N / 2}^{N-1}(N-n)=N^{2} / 4+N / 2$.

The orthogonal vectors $\mathbf{r}^{(, .)}$defined in Fig.1 are generators for $\mathscr{R}$, and $\mathscr{R}$ and $\mathscr{W}$ are isomorphic via $w$. This yields the next result.

Corollary 1. Let $\mathbf{x}$ and $\mathbf{y}$ be $N$-sample real-valued signals. Let $\mathrm{W}_{\mathrm{xy}}$ be the cross-WD defined by extending (1) to the correlation function $\mathrm{R}_{\mathrm{xy}}[n, m]=\mathrm{x}[n+m] \mathrm{y}^{*}[n-m]$. The following orthogonal vectors are generators for $\mathcal{W}$. $\mathbf{w}^{(n, k)}=\mathbf{w}_{\Delta^{(n+k)} \Delta_{\Delta^{(n-k)}}},(n+k, n-k) \in\{0, \ldots, N-1\}^{2}$, where $\Delta^{\left(n_{0}\right)}(n)$ denotes the pulse defined by $\Delta^{\left(n_{0}\right)}(n)=1$ if

\footnotetext{
${ }^{3}$ int $\{a\}$ denotes the integer part of $a$.
}

$n=n_{0}, 0$ otherwise. From the TF expansion coefficients ${ }^{4}$ $\alpha_{\mathrm{x}}^{(n, k)}=\left\langle\mathbf{w}_{\mathrm{x}} ; \mathbf{w}^{(n, k)}\right\rangle,(n+k, n-k) \in\{0, \ldots, N-1\}^{2},(3)$ the WD can be reconstructed according to $\mathbf{w}_{\mathrm{x}}=\sum_{(n, k)} \alpha_{\mathrm{x}}^{(n, k)} \mathbf{w}^{(n, k)},(n+k, n-k) \in\{0, \ldots, N-1\}^{2}$

Proof. It suffices to note that $\mathbf{r}^{(n, k)}=\mathbf{r}_{\Delta^{(n+k)}, \Delta^{(n-k)}},(n+k, n-k) \in\{0, \ldots, N-1\}^{2}$.

Since $w$ is an isomorphism of $\mathscr{R}$ into $\mathscr{W}$ that preserves orthogonality, it then follows that $w\left(\mathbf{r}^{(n, k)}\right)=\mathbf{w}^{(n, k)}$ are orthogonal generators for $\mathcal{Y N}$.

\section{CHARACTERIZATIONS OF $\mathscr{f}$ AND $\mathrm{L}_{\odot}$}

According to Theorem 1, information conveyed by the $N \times N$ discrete WD of any $N$-sample real-valued signal is redundant since every component $\mathrm{W}_{\mathrm{x}}[n, m]$ can be expressed as a linear combination of the $N^{2} / 4+N / 2$ elements of $\mathscr{\delta}$. The next theorem provides a necessary and sufficient condition satisfied by the elements of $\mathcal{S}$.

Theorem 2. Let $\mathbf{x}=(\mathrm{x}[0], \ldots, \mathrm{x}[N-1])^{T}$ be a $N$-sample real-valued signal with $N$ an even integer. Let $\mathcal{S}$ be a set of components $\mathrm{W}_{\mathrm{x}}[n, m]$ defined as

$\mathscr{S}=\bigcup_{n=0}^{\mathrm{N}-1} \mathscr{S}^{(n)}$,

with $\mathscr{S}^{(n)}=\left\{\mathrm{W}_{\mathrm{x}}[n, m]: m \in \mathcal{C M}^{(n)}\right\}$, $\mathcal{C M}^{(n)} \subset\{0, \ldots, N-1\}$ a set of $K^{(n)}+1$ indexes, $K^{(n)}=n$ if $n \in\{0, \ldots, N / 2-1\}, N-n-1$ otherwise.

Let $\mathbf{P}^{(n)}$ be the matrix whose $(k, m)$-component is

$\mathbf{P}^{(n)}[k, m]=\cos \left(\frac{4 \pi m k}{N}\right),(k, m) \in\{0, \ldots, N-1\}^{2}$.

Let $\mathbf{P}_{\delta}^{(n)}$ be the matrix obtained by extracting from $\mathbf{P}^{(n)}$ the first $K^{(n)}+1$ rows and the columns indexed by $\mathcal{C M}^{(n)}$.

The elements of $\&$ are generators for $\mathrm{W}_{\mathrm{x}}$ if and only if $\mathbf{P}_{\delta}^{(n)}$ is a nonsingular matrix, $\forall n \in\{0, \ldots, N-1\}$.

${ }^{4}<\mathbf{x} ; \mathbf{y}>$ denotes the inner product $\sum_{n} \mathrm{x}[n] \mathrm{y}^{*}[n]$. 

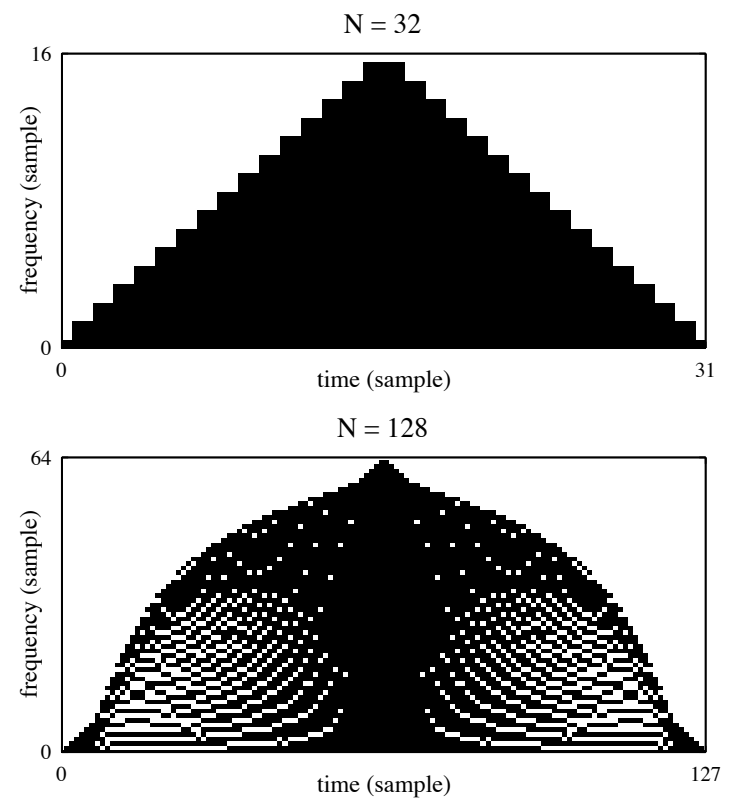

Figure 2. Examples of sets $\mathscr{S}$ of time-frequency generators $\mathrm{W}_{\mathrm{x}}[n, m]$ for $N=32,64,128$, and 256 (black areas).

Proof. Since $\mathrm{R}_{\mathrm{x}}[n,-k]=\mathrm{R}_{\mathrm{x}}[n, k]$, and $\mathrm{R}_{\mathrm{x}}[n, k]=0$ if $k>K^{(n)}$, we have

$\mathrm{W}_{\mathrm{x}}[n, m]=\mathrm{R}_{\mathrm{x}}[0,0]+2 \sum_{k=1}^{K^{(n)}} \mathrm{R}_{\mathrm{x}}[n, k] \cos \left(\frac{4 \pi k m}{N}\right)$.

The reader will see at once that it does not exist linear relations connecting the components $\mathrm{R}_{\mathrm{x}}[n, k], k \geq 0.5$ With (1) or (6), this implies that linear relations connecting TF components $\mathrm{W}_{\mathrm{x}}[n, m]$ are of the form $\sum_{m} a_{m} \mathrm{~W}_{\mathrm{x}}[n, m]=0$. This redundancy will be called spectral redundancy. Given $n$, let the discrete WD be expressed as $\mathbf{w}_{\mathrm{x}}^{(n)}=\mathbf{P}^{(n)} \mathbf{q}_{\mathrm{x}}^{(n)}$, with

$$
\begin{aligned}
& \mathbf{q}_{\mathrm{x}}^{(n)}=\left(\mathrm{R}_{\mathrm{x}}[n, 0], 2 \mathrm{R}_{\mathrm{x}}[n, 1], \ldots, 2 \mathrm{R}_{\mathrm{x}}\left[n, K^{(n)}\right]\right)^{T}, \\
& \mathbf{w}_{\mathrm{x}}^{(n)}=\left(\mathrm{W}_{\mathrm{x}}[n, 0], \ldots, \mathrm{W}_{\mathrm{x}}[n, N-1]\right)^{T} .
\end{aligned}
$$

Proceeding as in the proof of Theorem 1, one can show that $\operatorname{dim}\left\{\mathbf{q}_{\mathrm{x}}^{(n)}: \mathbf{x} \in \mathscr{X}\right\}=K^{(n)}+1$. It is also equal to $\operatorname{dim}\left\{\mathbf{w}_{\mathrm{x}}^{(n)}: \mathbf{x} \in \mathscr{\mathscr { X }}\right\}$ since the Fourier transform associates $\mathbf{w}_{\mathrm{x}}^{(n)}$ with $\mathbf{q}_{\mathrm{x}}^{(n)}{ }^{6}$. This means that every component of $\mathbf{w}_{\mathrm{x}}^{(n)}$ can be expressed as a linear combination of $K^{(n)}+1$ linearly-independent components [7, 8, 9]. Assume that $\mathcal{S}^{(n)}$ is such a set: $\mathscr{S}^{(n)}=\left\{\mathbf{w}_{\mathrm{x}}^{(n)}[m]: m \in \mathcal{C} \mathcal{M}^{(n)}\right\}$, with $\mathcal{C M}^{(n)}$ a set of $K^{(n)}+1$ indexes. Let now the relation $\mathbf{w}_{\mathrm{x}}^{(n)}=\mathbf{P}^{(n)} \mathbf{q}_{\mathrm{x}}^{(n)}$ be rewritten in the form (7) by reordering, if necessary, the rows of $\mathbf{w}_{\mathrm{x}}^{(n)}$ and $\mathbf{P}^{(n)}$.

$\left(\begin{array}{c}\mathbf{W}_{\mathrm{x}, \delta}^{(n)} \\ \overline{\mathbf{W}}_{\mathrm{x}, \delta}^{(n)}\end{array}\right)=\left(\begin{array}{c}\mathbf{P}_{\delta}^{(n)} \\ \overline{\mathbf{P}}_{\delta}^{(n)}\end{array}\right) \mathbf{q}_{\mathrm{x}}^{(n)}$,

\footnotetext{
${ }^{5}$ The validity of this assertion depends on $\mathscr{X}$, e.g., it is false when $\mathscr{X}$ denotes the space of $N$-sample analytic signals [5].

${ }^{6}$ One can verify that $\sum_{n=0}^{N-1}\left(K^{(n)}+1\right)=N_{\mathscr{f}}$.
}
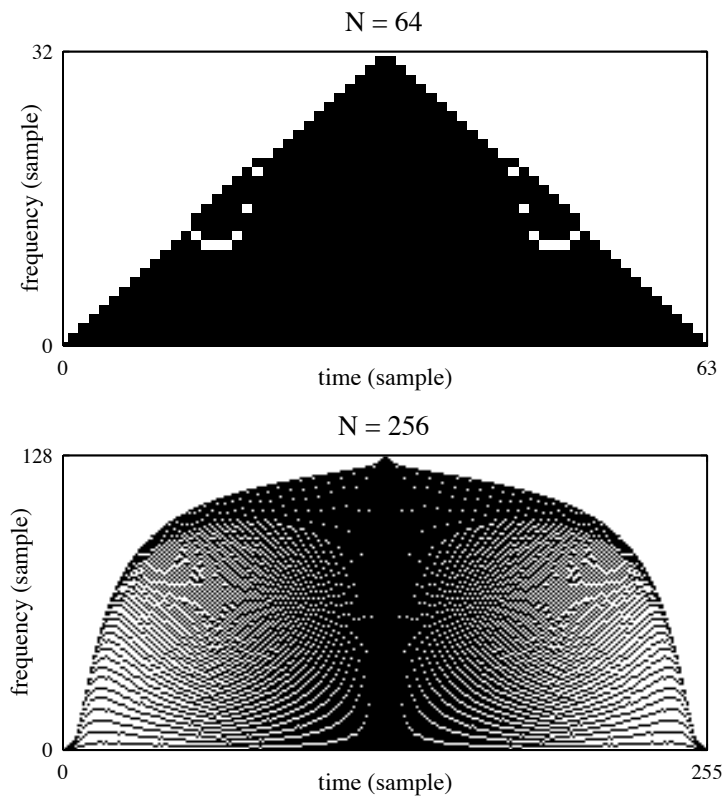

where $\mathbf{w}_{x, \mathcal{S}}^{(n)}$ is a vector whose $K^{(n)}+1$ components are the elements of the set $\mathscr{S}^{(n)}$. Then it follows that $\operatorname{dim}\left\{\mathbf{q}_{\mathrm{x}}^{(n)}: \mathbf{x} \in \mathscr{X}\right\}=\operatorname{dim}\left\{\mathbf{w}_{\mathrm{x}, \delta}^{(n)}: \mathbf{x} \in \mathscr{\mathscr { X }}\right\}=K^{(n)}+1$.

From the above we conclude that the matrix $\mathbf{P}_{\delta}^{(n)}$ satisfying $\mathbf{w}_{\mathrm{x}, \delta}^{(n)}=\mathbf{P}_{\delta}^{(n)} \mathbf{q}_{\mathrm{x}}^{(n)}$ is nonsingular [7, 8, 9].

Let $n$ be given, and let $\mathscr{S}^{(n)}=\left\{\mathrm{W}_{\mathrm{x}}[n, m]: m \in \mathcal{C} \mathcal{M}^{(n)}\right\}$ be a set of generators for the TF components $\mathrm{W}_{\mathrm{x}}[n, m]$, $m \in\{0, \ldots, N-1\}$. The following two properties are consequences of Theorem 2:

P1. The elements of $\left\{\mathrm{W}_{\mathrm{x}}[N-n-1, m]: m \in \mathcal{C} \mathcal{M}^{(n)}\right\}$ are generators for $\left\{\mathrm{W}_{\mathrm{x}}[N-n-1, m]: m \in\{0, \ldots, N-1\}\right\}$.

P2. The elements of $\left\{\mathrm{W}_{\mathrm{x}}[n, N / 2-m]: m \in \mathcal{C} \mathcal{M}^{(n)}\right\}$ are generators for $\left\{\mathrm{W}_{\mathrm{x}}[n, m]: m \in\{0, \ldots, N-1\}\right\}$.

Proof. Use successively the following relations to prove P1 and P2: $K^{(n)}=K^{(N-n-1)}, \mathbf{P}^{(n)}[k, m]=\mathbf{P}^{(n)}[k, N / 2-m]$.

We shall now characterize the linear relation $\mathrm{L}_{\delta}$ between components of $\mathrm{W}_{\mathrm{x}}$ and any set $\mathscr{\delta}$ of generators. Spectral redundancy of the discrete WD enables us to restrict our attention to linear maps $L_{\delta}^{(n)}$ that connect, given $n$, every $\mathrm{W}_{\mathrm{x}}[n, m]$ to any set $\mathscr{S}^{(n)}$ satisfying the conditions of Theorem 2.

Corollary 2. Assume that $\mathscr{S}^{(n)}=\left\{\mathbf{w}_{\mathrm{x}}^{(n)}[m]: m \in \mathcal{C} \mathscr{N}^{(n)}\right\}$ and $\overline{\mathcal{S}}^{(n)}=\left\{\mathbf{w}_{\mathrm{x}}^{(n)}[m]: m \in\{0, \ldots, N-1\} \backslash \mathcal{C} \mathscr{M}^{(n)}\right\}$ are sets of linearly independent and dependent components of $\mathbf{w}_{\mathrm{x}}^{(n)}$, respectively. Let $\mathbf{w}_{x, \&}^{(n)}$ and $\overline{\mathbf{w}}_{x, \delta}^{(n)}$ be vectors whose components are the elements of $\mathcal{S}^{(n)}$ and $\overline{\mathcal{S}}^{(n)}$, respectively. Let $\mathbf{P}_{\delta}^{(n)}$ and $\overline{\mathbf{P}}_{\delta}^{(n)}$ be the matrices obtained by extracting from $\mathbf{P}^{(n)}$ the first $K^{(n)}+1$ rows, and the columns indexed by $\mathcal{C M}^{(n)}$ and $\{0, \ldots, N-1\} \backslash \mathcal{C M}^{(n)}$, respectively. 
(a)

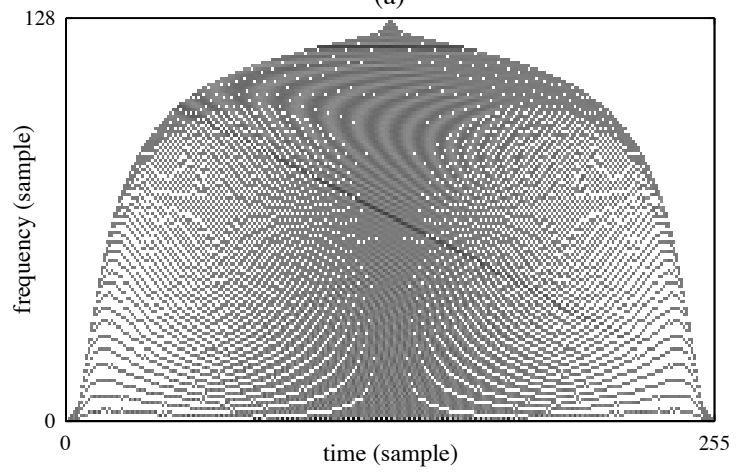

(b)

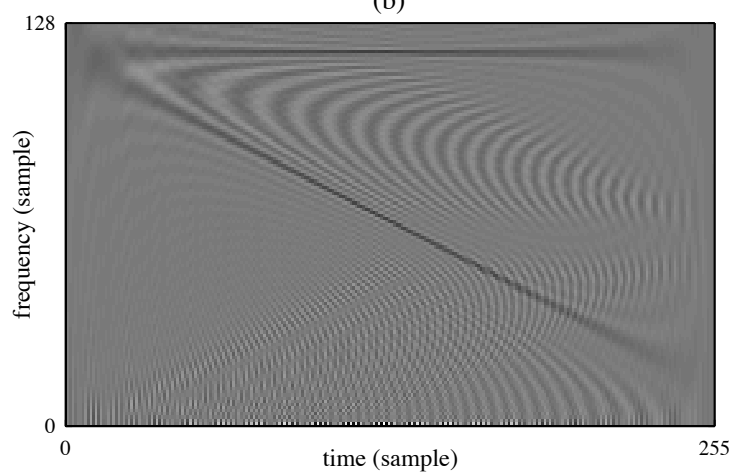

Figure 3.a. Nonredundant information conveyed by the WD of a 256-sample real-valued signal, here the sum of two chirps. Figure 3.b. WD reconstructed from nonredundant information represented previously.

The matrix $\mathbf{L}_{s}^{(n)}$ associated with the linear map $\mathrm{L}_{s}^{(n)}$ satisfying $\mathrm{L}_{\delta}^{(n)}\left(\mathbf{w}_{\mathrm{x}, \delta}^{(n)}\right)=\overline{\mathbf{w}}_{\mathrm{x}, \delta}^{(n)}$ is given by

$\mathbf{L}_{\delta}^{(n)}=\overline{\mathbf{P}}_{\delta}^{(n)}\left(\mathbf{P}_{\delta}^{(n)}\right)^{-1}$.

Proof. By (7), it follows that $\overline{\mathbf{W}}_{\mathrm{x}, \delta}^{(n)}=\overline{\mathbf{P}}_{\delta}^{(n)} \mathbf{q}_{\mathrm{x}}^{(n)}=\overline{\mathbf{P}}_{\delta}^{(n)}\left(\mathbf{P}_{\delta}^{(n)}\right)^{-1} \mathbf{W}_{\mathrm{x}, \delta}^{(n)}$.

\section{SIMULATION EXAMPLES}

Emphasis is now placed on computer simulations to illustrate the main theoretical results presented in previous sections.

In Figure 2, the black colored areas are examples of sets $\delta$ of $N^{2} / 4+N / 2$ TF generators $\mathrm{W}_{\mathrm{x}}[n, m]$ for $N=32,64$, 128 , and 256 . This means that information conveyed by white colored areas can be derived from information conveyed by black colored one using a linear transformation $\mathrm{L}_{\mathcal{\delta}}$. These sets of generators were found as follows. Let $n$ be given, and let $\mathbf{P}^{(n, m)}$ be the matrix obtained by extracting from $\mathbf{P}^{(n)}$ defined in (5) the first $K^{(n)}+1$ rows and the first $m$ columns. Let $\rho\left(\mathbf{P}^{(n, m)}\right)$ be the rank of $\mathbf{P}^{(n, m)}$. Each subset $\mathcal{S}^{(n)}$ such that $\mathcal{O}^{\prime}=\bigcup_{n} \mathcal{S}^{(n)}$ was determined by analyzing the variations of $\rho\left(\mathbf{P}^{(n, m)}\right)$ as a function of the variable $m, \quad m \in\left\{0, \ldots, K^{(n)}\right\}$ : $\rho\left(\mathbf{P}^{(n, m)}\right)>\rho\left(\mathbf{P}^{(n, m-1)}\right) \quad$ means that $\quad \mathrm{W}_{\mathrm{x}}[n, m] \in \mathcal{S}^{(n)}$. Finally, each matrix $\mathbf{L}_{\delta}^{(n)}$ associated with $\mathscr{S}^{(n)}$ was computed using (9). There we used to generate the discrete WD represented in Figure 3.b from nonredundant information conveyed by the generators presented in Figure 3.a.

\section{CONCLUSION}

In this paper, we have extended the works $[5,6]$ on the redundancy of the discrete WD by an analytic characterization of sets $\mathcal{S}$ of $\mathrm{TF}$ generators, and by studying some of their properties. Then we have characterized the linear map that connects these generators to every TF component.
Our results can be extended in the following directions:

- All results can be easily reformulated for the discrete ambiguity function.

- Redundancy of other representations of Cohen's class can be studied.

- This work can be extended to other linear signal spaces $\mathscr{X}$ such as discrete-time complex-valued and analytic signal spaces.

- These results were exploited to efficiently compute TF-based detectors $[6,7]$. However, there are other potentially interesting applications such as interference terms suppression, denoising, fast algorithms, etc. They can also certainly play a role in the resolution of signal synthesis problems.

\section{REFERENCES}

[1] Cohen, L., Time-frequency analysis. Prentice Hall, Englewood Cliffs, NJ, 1995.

[2] Flandrin, P., Time-frequency/time-scale analysis. Academic Press, San Diego, CA, 1999.

[3] Hlawatsch, F., Time-frequency analysis and synthesis of linear signal space, Kluwer Academic Press, Norwell, MA, 1998.

[4] Mecklenbräuker, W., Hlawatsch, F., editors, The Wigner distribution: theory and applications in signal processing, Elsevier, Amsterdam, The Netherlands, 1997.

[5] Richard, C., "Une méthodologie pour la détection à structure imposée. Applications au plan temps-fréquence". Ph.D. thesis, Compiègne University of Technology, 1998. http: //www-isis.enst.fr/Kiosque/theses /

[6] Richard, C., Lengellé, R. "On the dimension of the discrete Wigner-Ville transform range space". IEEE International Symposium on Time-Frequency and Time-Scale Analysis. Pittsburgh, October 1998.

[7] Lang, S., Algebra, Addison-Wesley, Reading, MA, 1994.

[8] Golub, G., Van Loan, C., Matrix computations, John Hopkins University Press, Baltimore, MD, 1993.

[9] Horn, R., Johnson, C., Topics in matrix analysis, Cambridge University Press, New York, NY, 1994. 